Maj. Marjorie Bolton stated that the choice of the continuation treatınent of these partially drug-resistant cases presented a difficult problem. Was it desirable to try the effect of an average course of treatment with several sulphonamides, or was it more suitable to give larger amounts of one of these compounds in order to obtain a higher concentration in the blood ? It was by no means a simple matter to decide when these cases should be classified as drug-resistant.

Dr. R. Marinkovitch asked Dr. Harkness whether there was any predominance of sex in the resistant cases. In his experience the majority of infections in women became partially resistant. In treating men he combined urethrovesical irrigations with chemotherapy and gave two tablets of sulphathiazole four times a day for five days. The irrigations were carried out for from two to four weeks. As soon as the urine became clear prostatic massage was done as a routine. He had not found the so-called true resistance to sulphathiazole.

Dr. R. Forgan said that he did not propose to follow Dr. Hanschell into the realms of clinical experiment which he had bravely entered, but he wished to refer to the fact that in America it had been possible to test the value of penicillin in cases of gonorrhoea resistant to sulphonamides. He thought it was not right that venereologists in Great Britain should be at a disadvantage compared with their colleagues in America in respect of a new chemical substance which, after all, had its origin in England. The Society was fortunate in having present the chief advisers on venereal diseases to the Navy, Army and Air Force. He suggested that the meeting should ask them to bring pressure on the authorities to release penicillin for experimental use by Dr. Harkness and other leading British venereologists.

Dr. F. C. Doble pointed out that some persistent infections responded to the injection of 5 to 15 cubic centimetres of whole blood. He quoted a case of stricture with repeated relapses of urethral discharge which illustrated the efficacy of this measure.

Col. L. W. Harrison (who was unable to be present), sent the following note.

"In case nobody taking part in the discussion has referred to some recent work by Kimmig, perhaps the following note may be interesting, as it is evidence that sulphonamide resistance is not likely to be acquired by under-dosage, as so many of us have feared, but is a natural attribute of certain strains of gonococci-perhaps because they have no appetite for sulphonamides. J. Kimmig (Klin. Wschr., 1943, 22, 31) trained four strains of gonococci which were normally sensitive to sulphonamides to become resistant in vitro, so that they were then able to grow in a concentration of 1 in 60,000 . At this stage they were inoculated into human urethras but in repeated trials completely failed to infect. In contrast, the parent strains of two of these artificially resistant strains. were found to be still virulent when they were in their seventieth subculture, showing that the loss of virulence in the sulphonamide-resistant offspring was not due to age in culture. All attempts to revive the virulence of these artificially resistant strains failed. Kimmig isolated from sulphonamide-resistant cases of gonorrhoea eight strains, of which five were able to grow in medium containing concentrations of sulphonamide as strong as 1 in 2,000 and three in a concentration of 1 in 20,000 . I suggest that the explanation is that they had no appetite for the sulphonamide so that it was unable to bring about their starvation."

Dr. Harkness, in reply, said he did not consider that the incidence of drug resistance was higher in the female than in the male. The female was more liable to develop closed foci of infection which were often resistant to the sulphonamides, and surgical interference might be necessary. The treatment for drug resistance, partial or complete, was the same; the best method for observing progress was to make an examination before the first morning micturition. He agreed with Wg. cdr. McElligott that complete and partial drug resistance were not always clear-cut entities, but he could not understand why Wg. cdr. McElligott had not observed the persistence of profuse gonococcal discharges when the resistance was complete : this was much more frequent than partial resistance. In reply to Dr. Marinkovitch he considered that the posterior urethra was always involved in these cases and that sulphathiazole was the drug of choice. Dr. Marinkovitch had made an interesting observation concerning the incidence of residual infections due to non-specific organisms. These cases were mostly due to a faulty technique in irrigation but they sometimes occurred in clinics where urethrovesical irrigations were not prescribed until a diagnosis of drug resistance had been made. Dr. Harkness considered that the primary infection in these cases was caused by the gonococcus and one or more non-specific organisms. Dr. Hanschell had made an interesting observation about intravenous injections of propamidine. He, Dr. Harkness, was also trying $p$-chloro-xylenol in 70 per cent solution of methyl acetamide (as recommended by Zondeck and Bromberg). and the sodium salts of pentose nucleotides. He had not yet observed the effects of these drugs on a sufficient number of cases to form a definite opinion on their efficacy.

\title{
The Wassermann reaction
}

The problem of carrying out the Wassermann reaction without the guinea-pig complement has been solved by the serological laboratory of the Leningrad Institute for Blood Transfusion. Fresh blood serum is substituted ; in about 70 per cent of cases active complement occurs in human blood. Some 50,000 tests have already been made by means of the new method and they prove that it is possible to employ the human complement when carrying out the Wassermann reaction. The remnants of the sera of the preceding experiments have now been found to be usable. This method reduces the cost and simplifies the procedure of carrying out this reaction.-U.S.S.R. Society for cultural relations with other countries, Medical Chronicle, January, 1944. 